\title{
Las editoriales independientes frente al problema de la distribución: los casos de Eterna Cadencia y Santiago Arcos
}

\author{
Gustavo Daniel Velázquez \\ Departamento de Ciencias Sociales, Universidad Nacional de Quilmes. \\ gdvelazquez90@gmail.com \\ Fecha de finalización del trabajo: 1 de marzo de 2020. \\ Recibido: 5 de marzo de 2020. \\ Aceptado: 20 de mayo de 2020 . \\ DOI: https://doi.org/10.26422/aucom.2020.0902.vel
}

\section{Resumen}

El objetivo de este trabajo es reflexionar sobre la distribución editorial desde la perspectiva de las editoriales independientes. Estas editoriales de pequeña y mediana escala económica encuentran desafíos para dar solución a la circulación comercial de sus publicaciones. Algunos editores recurren a la contratación de empresas de distribución y otros deciden encarar la tarea por cuenta propia. Ambos métodos poseen sus propias particularidades, los cuales se pretenden analizar en el marco de este artículo. Con este fin, se abordan las experiencias de las editoriales Eterna Cadencia y Santiago Arcos.

Palabras clave: industria del libro, distribución editorial, editoriales independientes.

\section{Independent publishing houses and the problem of distribution: the cases of Eterna Cadencia and Santiago Arcos}

\begin{abstract}
In this paper, we will consider editorial distribution from the point of view of independent publishing houses. These small and mid-range publishers face unique challenges when it comes to commercially distributing their book releases. Some publishers hire specialized distribution companies, while others take on this task themselves. Both methods present their own quirks, which we analyze in what follows. To this end, we will discuss the experiences of two independent publishers in Argentina, Eterna Cadencia and Santiago Arcos.

Keywords: book publishing industry, editorial distribution, independent publishers.

\section{Editoras independentes que enfrentam o problema da distribuição: os casos da Eterna Cadencia e Santiago Arcos \\ Sumário}

O objetivo deste trabalho é refletir sobre a distribuição editorial da perspectiva de editoras independentes. Esses editores de pequena e média escala enfrentam desafios para resolver a cir-
\end{abstract}


Gustavo Daniel Velázquez

Las editoriales independientes frente al problema de la distribución: Ios casos de Eterna Cadencia y Santiago Arcos

culação comercial de suas publicações. Alguns editores recorrem ao recrutamento de empresas de distribuição e outros decidem executar a tarefa por conta própria. Ambos os métodos têm suas próprias particularidades, que devem ser analisadas na estrutura deste artigo. Para isso, são discutidas as experiências das editoras Eterna Cadencia e Santiago Arcos.

Palavras chave: indústria de livros, distribuição, editoras independentes.

\section{Introducción ${ }^{1}$}

La fase de distribución de la cadena de valor de la industria editorial es un aspecto fundamental para la circulación y el acceso a los libros. Esta tarea determina el comercio de los ejemplares a partir de la gestión articulada con los puntos de venta, de manera que dicha actividad influye en la economía de las editoriales, dado que las ventas son la principal fuente de financiamiento de estas empresas.

Los sellos que desean intervenir en el mercado deben definir una estrategia para dar respuesta a este aspecto de la industria editorial, pues existen distintas formas de encarar la distribución. Este trabajo puede tercerizarse o, incluso, realizarse por cuenta propia.

Por un lado, el método de distribución tercerizada supone la participación de un agente distribuidor encargado exclusivamente de la tarea de circulación comercial. Por otro lado, a través de la autodistribución, los editores encaran la tarea de logística por cuenta propia, haciéndose cargo de los costos y del trabajo necesario.

Ambas formas de distribución presentan determinados desafíos para las editoriales independientes. Este conjunto de emprendimientos de pequeña y mediana escala financiera representan un segmento de importancia dentro del mercado nacional. ${ }^{2}$ Específicamente, las editoriales independientes se desenvuelven con base en márgenes productivos acotados (es decir, números reducidos de novedades) y tiradas pequeñas (Botto, 2006). De modo que los márgenes productivos y comerciales de este tipo de proyectos generan tensión al momento de definir la distribución, una actividad que depende en gran medida de las posibilidades financieras de cada sello.

Este trabajo, por tanto, pretende ser una aproximación -caracterización y análisis- al tema de la distribución, con especial énfasis en el modo en cómo las editoriales independientes resuelven dicha cuestión. Para esto, se toman como ejemplos las experiencias de las editoriales Eterna Cadencia y Santiago Arcos. Estos casos servirán para

\footnotetext{
El trabajo pertenece al Proyecto de investigación "La edición en la era de redes. Entre el artesanado y las tecnologías digitales".

Según el relevamiento llevado a cabo por la Universidad Nacional de Quilmes, sistematizado por Daniel Badenes, de 426 editoriales independientes, el 92,5\% nació en los últimos años, especialmente durante el quinquenio 20112015, período en el cual se lanzaron cuatro de cada diez editoriales existentes en la actualidad. Asimismo, durante la presidencia de Mauricio Macri, no se detuvo el fenómeno de surgimiento de nuevas editoriales: "Un centenar de sellos independientes corresponden a estos últimos cuatro años de gobierno, es decir que pese al saqueo en el plano económico y al vaciamiento de las políticas culturales, siguieron gestándose proyectos en la Argentina, a razón de dos por mes" (Badenes, 2019, p. 21).
} 
pensar en las ventajas y dificultades tanto de la distribución tercerizada como de la autodistribución,

Eterna Cadencia es una editorial creada en 2008. Este sello, abocado a la narrativa y al ensayo, publica tanto a autores nacionales como internacionales, contemporáneos y clásicos. A través de una distribuidora externa, las producciones de la editorial llegan a toda Argentina, también a países de América Latina -como Chile, Colombia, Uruguay, Perú y México- y a España.

Santiago Arcos es una editorial que comenzó a funcionar en 2002 y se enfoca en la publicación de libros de ficción y ensayos. Respecto a la distribución, el editor Miguel Villafañe realiza la tarea por cuenta propia. Distribuye directamente a librerías de la Ciudad Autónoma de Buenos Aires, principalmente, y a algunas librerías particulares de Mar del Plata, Mendoza y Rosario.

\title{
Marco teórico
}

Este artículo parte de la consideración de que la distribución constituye un aspecto que no ha sido abordado de manera exhaustiva por los estudios del libro y la edición. Por tanto, la información que aquí se brinda es, en gran medida, una reconstrucción a partir de los testimonios relevados de editores (sobre esto se volverá en el siguiente apartado).

De tal manera, se toman los aportes de la economía política de la comunicación (EPC) para pensar este objeto de estudio. Como plantea Vicente Mosco (2006), la EPC se entiende como el "estudio de las relaciones sociales, particularmente las relaciones de poder, que mutuamente constituyen la producción, distribución y consumo de recursos, incluidos los recursos de comunicación" (Mosco, 2006, p. 59).

Esta perspectiva de análisis resulta adecuada para abordar el objeto de estudio, especialmente respecto a los análisis específicos de las industrias culturales. En este sentido, se toma la definición de Gaëtan Tremblay (2011), quien considera a las industrias culturales como

\begin{abstract}
El conjunto en constante evolución de las actividades de producción y de intercambios culturales sometidas a las reglas de la comercialización, donde las técnicas de producción industrial son más o menos desarrolladas, pero donde el trabajo se organiza cada vez más en el modelo capitalista de una doble separación entre el productor y el producto, entre las tareas de creación y ejecución. De este doble proceso de separación resulta una pérdida creciente del control de los trabajadores y artistas sobre el producto de su actividad (p. 115).
\end{abstract}

La industria editorial se inscribe en este conjunto de actividades abocadas a la producción y circulación de bienes simbólicos. Adquiere, por tanto, las particularidades propias de las actividades dedicadas a la economía de recursos culturales: importancia del trabajo creativo, doble dimensión de productos -económica y simbólica-, exigen- 
Gustavo Daniel Velázquez

Las editoriales independientes frente al problema de la distribución: Ios casos de Eterna Cadencia y Santiago Arcos

cia de renovación constante de productos, incertidumbre sobre los resultados y carácter aleatorio de la demanda (Zallo, 1988).

También se considera importante destacar la tendencia a la concentración dentro de las ramas de las industrias culturales. Específicamente, en este artículo se adopta la postura crítica que ve la concentración como un fenómeno que puede resultar negativo para el mercado y para la sociedad en su conjunto, pues la posición dominante de los grandes grupos concentrados puede generar barreras de entrada para nuevos competidores y atentar contra la pluralidad y el pluralismo (Becerra y Mastrini, 2009; 2017).

Asimismo, en este trabajo se recuperan distintos aportes del campo de estudios sobre el libro y la edición, particularmente de los referentes Alejandro Dujovne (2017; 2018), José Luis de Diego (2006; 2015), Daniela Szpilbarg $(2010 ; 2013 ; 2019)$ y Ezequiel Saferstein $(2013 ; 2019)$. Estos investigadores se han preocupado por reflexionar sobre el desarrollo de la industria editorial nacional, en especial sobre los procesos de concentración en el mercado editorial desde la década del 90, las políticas editoriales de los grupos concentrados y la edición independiente en el país.

A su vez, se considera importante la idea expuesta por Pierre Bourdieu (2000) acerca de la mercantilización que atraviesa el campo editorial, en el cual las normas comerciales, impuestas por los grupos concentrados, transforman las prácticas editoriales. En este sentido, el autor considera a los pequeños editores como "el bastión central de la resistencia a las fuerzas de mercado" (Bourdieu, 2000, p. 263).

En lo que respecta a la edición independiente, también resultan relevantes los aportes de Gilleu Colleu (2008), quien considera a las editoriales independientes como proyectos al margen de los dictados meramente comerciales de la edición que proponen los grandes conglomerados.

\section{Metodología}

Este trabajo es un avance de la tesis de Maestría en Industrias Culturales (UNQ) Letra chica. Producción, distribución y comercialización de las editoriales independientes (2011-2015), ${ }^{3}$ la cual se encuentra en desarrollo. En ella, se parte del relevamiento de experiencias de editoriales independientes de la Ciudad Autónoma de Buenos Aires a partir de un estudio de caso múltiple. Por tanto, aquí se toma un pequeño recorte de los casos estudiados.

Este artículo, entonces, está sustentado en una estrategia metodológica cualitativa para analizar e interpretar el objeto de estudio, basado principalmente en entrevistas

\footnotetext{
La invesigación correspondiente a la tesis se sustenta en la experiencia de dieciocho editoriales: Ediciones del Signo, Ediciones Godot, Lenguaje Claro, Eterna Cadencia, Grupo Editorial Sur, Gourmet Musical, Biblos, Peces de Ciudad, Alto Pogo, Marea Editorial, Nulú Bonsai, Santiago Arcos, Las Cuarenta, Chirimbote, Punto de Encuentro, Entropía, CICCUS y Patria Grande. Estos casos fueron seleccionados procurando obtener la mayor diversidad posible (temáticas, públicos, trayectoria, alianzas) para enriquecer el trabajo.
} 
Austral Comunicación

Volumen 9, número 2 (Diciembre de 2020): 405-422. ISSN 2313-9129

semiestructuradas y en profundidad a editores de sellos independientes, realizadas en noviembre de 2017. En esta tarea, se pretendió relevar la experiencia de estos actores respecto a la manera en cómo resuelven distintos aspectos de la rutina productiva editorial. En lo que se refiere a la distribución mayorista, las ventajas, problemáticas y desafíos que enfrentan en estos tiempos en la cadena de valor.

Para ello, se les realizaron entrevistas a Leonora Djament (editora de Eterna Cadencia) y Miguel Villafañe (editor de Santiago Arcos). Además de abocarse a las acciones editoriales, ambos editores ejercen un rol activo en la parte comercial de sus proyectos.

Estas editoriales fueron elegidas por las distintas experiencias que cada una posee respecto a la distribución, además de la similitud en la dimensión económica de ambos proyectos (estos sellos pueden definirse como empresas de mediana escala). Por un lado, Eterna Cadencia trabaja con una distribuidora externa, tanto para el posicionamiento de los libros en el plano nacional como internacional. Por otro lado, Santiago Arcos encara la distribución por cuenta propia, generando así un circuito propio de circulación para sus publicaciones.

Ambos sellos sostienen sus respectivos métodos de distribución desde sus comienzos, por lo que la comparación de estos casos servirá para determinar los puntos fuertes y los débiles de cada sistema de circulación comercial.

La información obtenida de esta instancia es acompañada de datos estadísticos pertenecientes al Informe de producción del libro argentino, confeccionado por la Cámara Argentina del Libro, además de bibliografía especializada para una reflexión más acabada del objeto de estudio.

Dado que los casos de análisis de este artículo pueden ubicarse dentro del conjunto de las llamadas editoriales independientes, resulta necesario destacar que se considera al sector "independiente" como una esfera ampliamente heterogénea en la que conviven diferentes tipos de propuestas editoriales (Vanoli y Saferstein, 2011). Esta diversidad de proyectos editoriales se debe a las escalas económicas, los modelos de negocio, las temáticas que componen el catálogo y la dimensión política de la propuesta editorial, entre otras variables.

Este tipo de sellos se diferencian de las grandes empresas por su menor estructura económica y financiera, cuya posición en el mercado se define por el cuidado de los catálogos. Muchas de estas editoriales chicas han evolucionado hasta llegar a una etapa de profesionalización (Badenes, 2019), en la que tienen amplio conocimiento del funcionamiento del mercado del libro y enfrentan el desafío de fortalecer los aspectos productivos y comerciales.

Dentro de este sector, también caben las editoriales artesanales, las cuales se caracterizan por poseer sus propios talleres en donde imprimen y encuadernan (Schierloh, 2019). De esta forma, trabajan sobre la base de tiradas pequeñas y distribución en espacios no convencionales (Conde y Hafter, 2019). 
Gustavo Daniel Velázquez

Las editoriales independientes frente al problema de la distribución: los casos de Eterna Cadencia y Santiago Arcos

Cabe mencionar que la categoría "independiente", a su vez, genera adhesiones y rechazos entre los propios actores editoriales. Las imprecisiones de este término lleva a que se englobe un conjunto de propuestas editoriales de distinto signo: "Cada tanto se reitera la discusión acerca del sentido y función de la categoría 'independiente.' [...] La crítica a esa categoría tiene un punto fuerte: su imprecisión al momento de distinguir qué sellos entran ahí y cuáles no" (Dujovne, 2018).

Los casos de estudio de este artículo, por tanto, pueden ser identificados como editoriales de mediana escala (poseen al menos un trabajador asalariado), en las que el editor ejerce una tarea de filtrado de contenido (Bhaskar, 2014) y donde hay una preocupación por profesionalizar los procesos de trabajo en pos de llegar a la mayor cantidad posible de lectores y de autosustentarse.

\section{La distribución editorial como problema}

Ramón Zallo (1988) menciona los diferentes tipos de actividades de las industrias culturales, las cuales pueden clasificarse en: edición discontinua (incluye a las industrias cinematográficas, fonográficas y editoriales), edición continua (prensa gráfica) y emisión o difusión continua (industria de la radio y la televisión). Todas estas ramas se diferencian por el grado de industrialización capitalista, por el ciclo productivo que adopta (continuo o discontinuo), por el tipo sumisión del trabajo al capital y por el modo en que este lleva adelante los procesos de valorización y realización de las mercancías. En este sentido, entonces, el sector editorial está determinado por su funcionamiento discontinuo, lo cual se torna en una economía basada en stock (De Mateo Pérez, Saura y Casals, 2009).

La actividad editorial encierra los procesos de producción, distribución, comercialización y consumo (recepción) de publicaciones editoriales. De esta manera, el sector editorial se compone de una multiplicidad de oficios y especialidades. Los profesionales intervienen en diferentes momentos de la cadena de valor, desde el suministro de la materia prima hasta que el consumidor toma contacto con el producto.

$\mathrm{Al}$ respecto, la distribución se presenta como una etapa fundamental de la cadena de valor, puesto que encierra un conjunto de acciones que hacen a la circulación y al acceso al libro.

Esta actividad combina rasgos técnicos y logísticos que influyen decisivamente en el nivel de ventas, principal fuente de ingresos de las editoriales. De hecho, tanto Eterna Cadencia como Santiago Arcos financian sus publicaciones con los ingresos propios del proyecto editorial que, como manifiestan sus editores, provienen de las ventas. Por tanto, no es menor la incidencia que tiene la distribución comercial en la sostenibilidad de las empresas de edición. 
En este sentido, los editores optan por el método de distribución mayorista que mejor se adecue a las condiciones económicas del emprendimiento. Como plantean De Mateo Pérez, Saura y Casals (2009):

\begin{abstract}
La definición de estrategias de distribución parte de la fijación de objetivos de distribución - por ejemplo, mejorar la distribución para determinados segmentos, diversificar puntos de venta o fidelizar clientes, entre otros-. A partir de ahí se establece el modelo de distribución y los medios técnicos necesarios, y se elabora el presupuesto de distribución. Se definen también los controles o indicadores de evaluación de la calidad del servicio de distribución, que permitirán ir revisando la estrategia una vez que se entre en la fase de ejecución (p. 126).
\end{abstract}

Particularmente, las formas principales en que esta tarea se lleva adelante son: a través de la distribución externa (es decir, contratando a empresas que brindan un servicio de distribución de libros) o apelando a la distribución propia (autodistribución). Cada sistema posee su grado de dificultad de acuerdo con el esquema de mediación que supone, ${ }^{4}$ generando una serie de ventajas y desventajas para los editores independientes.

Cabe mencionar que las editoriales independientes afrontan distintos desafíos propios de la escala económica de los proyectos, como la obtención de financiamiento para solventar sus publicaciones y la consecuente dificultad para volverse emprendimientos sostenibles (Amadeo González, 2019). Una forma de distribución, sea cual fuere, requiere un nivel diferente de capital y trabajo, condición que pone en tensión a los proyectos de pequeña y mediana escala financiera.

Así, también deben lidiar con las complejidades propias de la estructura del mercado nacional, que posee altos niveles de concentración (De Diego, 2012; Paéz, 2018; Szpilbarg, 2019). Las características del mercado, dominado prácticamente por dos grandes grupos -Planeta y Penguin Random House (Dujovne, 2017)-, también generan condiciones desiguales de distribución para los proyectos independientes. Estos actores que se conforman a partir de la participación de los fondos de inversión en el negocio editorial (Colleu, 2008) y la internacionalización de los mercados (Jambeiro, Brittos y Simis, 2005) poseen mayor capacidad de negociación con distribuidores y libreros, aspecto que repercute en mejores exhibiciones de sus productos.

Tales variables afectan la economía particular de cada sello. Asimismo, a partir de estas condiciones, la distribución se liga con las posibilidades de acceso de los consumidores a determinadas ediciones. Si se toma en consideración que el libro posee una doble valoración -valor de mercado y valor simbólico (Zallo, 1988)-, no podemos desentendernos de la importancia que tiene la distribución para el acceso a dichos bienes culturales. Dado que esta actividad se presenta como un factor fundamental en

\footnotetext{
4 Cualquier sistema de distribución se complementa, en la mayoría de los casos, con la venta directa al lector, que se desarrolla en gran parte a través de ferias. La venta a través de una feria posee su propia logística y, por ende, complejidad. Por tanto, en este escrito no se abordan las particularidades de esta estrategia comercial.
} 
Gustavo Daniel Velázquez

Las editoriales independientes frente al problema de la distribución: Ios casos de Eterna Cadencia y Santiago Arcos

el recorrido que toma un libro desde que sale de la imprenta hasta que toma contacto con el lector (pasando por el librero u otros actores comerciales), no se puede obviar la trascendencia que tiene la distribución comercial para la circulación de ideas.

\section{Distribución tercerizada}

La forma tradicional de circulación comercial de los ejemplares es a través de la distribución tercerizada. Según la Cámara Argentina del Libro (2019), los distribuidores han adquirido importancia entre las pequeñas y medianas editoriales en los últimos cuatro años.

En términos generales, las principales tareas de un distribuidor son:

- Logística: almacenaje, recogida (picking), embalaje (packing), devoluciones, facturación y cobros.

- Comercialización: mercadeo, comercialización, promoción y servicios de pre y posventa (Gil y Gómez, 2017).

Tales acciones hacen que el distribuidor se constituya como un agente intermediario de importancia tanto para la editorial como para las librerías. El distribuidor recibe los ejemplares y se encarga del almacenamiento, preparación de pedidos, armado de cajas, gestión del transporte, verificación de las cuentas de los libreros y facturación. También se ocupa de administrar los libros que retornan de los puntos de ventas (Colleu, 2008).

Este agente trabaja con el catálogo de la editorial que lo contrató y se encarga de "posicionar" los libros en las librerías. Por tanto, se encuentra en contacto permanente con el librero, al cual provee de los materiales de trabajo.

Para las editoriales independientes, la distribución externa presenta determinadas ventajas. Principalmente, trabajar con una distribuidora tercerizada libera a la editorial de la necesidad de desarrollar una estructura de logística que resulta costosa y requiere la dedicación de personal abocado específicamente a dicha tarea. Así, los editores pueden desligarse de las acciones de gestión y administración que implica distribuir, tales como las cobranzas y los documentos correspondientes (facturas, remitos, etcétera).

\footnotetext{
Supongo que hay algo del quehacer cotidiano donde estamos un poquito menos encima de lo que estaríamos si la distribución fuera propia. Por supuesto, todo el tema de la cobranza nos preocupa, porque si no se le paga a nuestro distribuidor, el problema repercute en nosotros, pero bueno, no es el estrés de estar día a día tratando de cobrar (Leonora Djament, editora de Eterna Cadencia). ${ }^{5}$
}

Eterna Cadencia distribuye en todo país a través de Waldhuter. Como menciona la editora Leonora Djament, dejar la circulación mayorista a una empresa dedicada a esta

Entrevista realizada el 22 de noviembre de 2017 
cuestión exime a la estructura editorial de la mayor parte de las actividades administrativas, principalmente de la cobranza de las librerías, tarea que insume mucho tiempo.

Además de Djament, quien desde la Dirección Editorial se ocupa de la parte comercial y editorial, Eterna Cadencia cuenta con cuatro integrantes que trabajan a tiempo completo: una persona en prensa, otra en producción y edición y una asistente en comercialización y comercio exterior. Además, una persona que trabaja a medio tiempo se encarga de derechos de autor y hay un equipo de colaboradores freelance. De modo que la empresa cuenta con una estructura organizada, en la cual existe un área particular para atender las cuestiones de mercado.

Se puede verificar, entonces, la importancia que este sello le brinda al aspecto comercial. ${ }^{6}$ De hecho, Eterna Cadencia se constituyó primeramente como una librería y, más tarde, por decisión de su fundador, Pablo Braun, sumó su propia editorial. Este recorrido es igual al de la editorial Santiago Arcos, la cual gestiona su propia librería a la par de la edición de libros.

Otra ventaja importante que brindan las distribuidoras mayoristas es el ingreso a muchas librerías. Esto es valorado por los sellos independientes, dado que agiliza la presencia de sus catálogos, sobre todo en diferentes provincias del país: “Tal vez, cuando se empieza y las librerías no te quieren tomar los libros, está bien trabajar con un distribuidor, porque él te coloca los libros" (Miguel Villafañe, editor de Santiago Arcos).

Como bien menciona Villafañe, en los inicios de muchos proyectos editoriales puede resultar dificultoso lograr que un librero tome libros de un sello que apenas conoce. Ante este problema, una persona especializada en la materia, como lo es un distribuidor, puede ayudar a la colocación de los libros en los puntos de venta.

Tanto es así que existe una marcada diferencia en la cantidad de librerías con las que trabaja cada sello: Santiago Arcos distribuye en cuarenta librerías y Eterna Cadencia llega a más de cien. ${ }^{7}$ La estructura con la que cuenta Santiago Arcos, de alguna manera, le impide tener presencia en más locales de venta. Como se verá más adelante, el equipo que integra el sello es reducido, por lo que con la autodistribución solo es posible llegar a una cantidad escasa de locales.

\footnotetext{
6 En esta misma línea, en 2019, el fundador de Eterna Cadencia lanzó la distribuidora Big Sur. Esta plataforma nace para fomentar la distribución de las editoriales independientes entre Argentina, Chile, Uruguay, Venezuela, México y España (big-sur.net). Dicha propuesta se une al conjunto de distribuidoras nacidas de editoriales independientes, tales como Malisia, Carbono o La Coop. A diferencia de Big Sur, estas son, ante todo, agrupaciones de editoriales que se orientan a resolver problemas comunes de manera conjunta, en lo que habitualmente la distribución se presenta como el principal desafío. La ventaja de la distribución asociada consiste en alivianar costos y trabajo entre todos los miembros. Para más información sobre estas distribuidoras, ver Velázquez (2019).

Tanto Santiago Arcos como Eterna Cadencia dejan sus libros en consignación. Este sistema consiste en "la entrega por parte de las editoriales de una cantidad de libros a las librerías para que los vendan, con el compromiso de declarar (en forma mensual) las ventas efectivamente realizadas" (Centro de Estudios para la Producción, 2014, p. $58)$.
} 
Gustavo Daniel Velázquez

Las editoriales independientes frente al problema de la distribución: Ios casos de Eterna Cadencia y Santiago Arcos

La distribución por terceros, entonces, ofrece condiciones favorables para la ampliación de la visibilización y comercialización de libros. Al respecto, también debe tenerse en cuenta la circulación a nivel internacional. Eterna Cadencia tiene presencia en muchos países. Para ello, trabaja con distribuidores del exterior, como Siglo del Hombre, Fernández de Castro Libros, UDL Libros, Sophos, Almadía, La Familia y Escaramuza SRL. Esto le permite que su catálogo circule en mercados internacionales. Santiago Arcos, en cambio, no posee distribución mayorista en el exterior.

Por otra parte, la distribución a través de terceros puede generar ciertas dificultades para los pequeños y medianos sellos. Así lo expresa Villafañe:

\begin{abstract}
Acá, se le entregan los libros al distribuidor y él los desparrama. Después, le pedís la devolución de libros y no lo hace porque hay cinco en Jujuy, dos en Bariloche, tres en Salta, dos en Santa Fe, cinco en Mendoza. En total son cien libros y el distribuidor no se hace cargo de ellos porque dice que no le da el costo del flete, que, en efecto, es altísimo. Entonces, quedan los libros varados por ahí, no vuelven nunca. En general, decís "bueno, son cien libros", pero te estoy hablando de quinientos ejemplares, que representan el $20 \%$ de la tirada La ganancia neta de una editorial es del 6,5 al 7\%. Si perdés cien ejemplares, perdiste mucho más que la ganancia (Miguel Villafañe, editor de Santiago Arcos). ${ }^{8}$
\end{abstract}

Además, agrega que dado que las editoriales independientes se manejan con una base de tiradas chicas (la tirada promedio de este sello es de quinientos ejemplares), destinar la mayor parte a un distribuidor representa una gran apuesta. A su vez, los tiempos propios de la distribución se tornan largos, repercutiendo en las liquidaciones provenientes de las ventas que llegan a la editorial varios meses después de la declaración del librero.

En cuanto a esto, cabe aclarar que con el método tercerizado, cuando un librero necesita reponer títulos, los pide al distribuidor, quien, a su vez, se los solicita a la editorial. Este recorrido implica largos plazos de gestión. Los ejemplares que pidió la librería llegan al cabo de meses, haciendo que se congele la demanda del título en cuestión (López Winne y Malumián, 2016).

Sobre este punto, Djament considera que:

[La desventaja de la distribución a través de terceros radica en] no tener la posibilidad de realizar una acción directa e inmediata en el punto de venta, sino que hay que pasar por el distribuidor. Si necesitamos recuperar libros que están consignados en las librerías, se recuperan en un tiempo que no es el que desearíamos. De las ventas nos enteramos un poco más tarde, porque primero las conoce el distribuidor (Leonora Djament, editora de Eterna Cadencia). ${ }^{9}$

Por tanto, los tiempos propios del circuito de distribución repercuten en las posibilidades comerciales. A este conflicto se le suma no poseer información específica de cada canal comercial, la cual, muchas veces, retienen las distribuidoras. Esto tiene que

\footnotetext{
8 Entrevista realizada el 15 de noviembre de 2017.
}

9 Entrevista realizada el 22 de noviembre de 2017. 
ver con desconocer cómo y dónde se encuentran los libros. ${ }^{10}$ Tales aspectos pueden generar caídas en las ventas, además de pérdida de ejemplares.

En otro orden, las distribuidoras mayoristas suelen acaparar gran cantidad de sellos, de modo que trabajan con varias firmas a la vez. En esta estrategia de financiamiento resulta común que los grandes grupos editoriales obtengan prioridad por parte de los distribuidores. La razón responde a que los libros de rápida circulación que estos publican generan más ingresos para el distribuidor y el librero.

En otras palabras, muchas veces los resultados de un servicio de distribución no son beneficiosos para las editoriales pequeñas, cuyos catálogos son de rotación lenta y requieren de tiempos largos de posicionamiento en el mercado. Los productos de los sellos independientes no funcionan de la misma manera que los libros de los grandes sellos, dado que son realizaciones orientadas a nichos de la demanda (Saferstein y Szpilbarg, 2014) que apuestan por la perdurabilidad del libro a lo largo del tiempo (longseller).

Las producciones de los grupos editoriales, en cambio, son impulsadas por el marketing (Bustamante, 2008) y las modas de éxito inmediato (Muniz Júnior, 2015). Por tanto, obtienen ventajas de los distribuidores y logran mejores acuerdos con las librerías.

Tales aspectos llevan a considerar que la distribución se vuelve un problema estructural que afecta de manera desigual a los distintos segmentos del mercado, pues la concentración como proceso de mercado lleva al acaparamiento de la distribución y exhibición/comercialización por parte de los grandes grupos económicos (Becerra y Mastrini, 2009).

De hecho, en el Informe de producción del libro argentino realizado por la Cámara Argentina del Libro (2018) se plantea un posible crecimiento de la presencia de las grandes casas editoriales en el canal librero. Debido a la reducción de las primeras tiradas de las pymes en ese sector, "cabe la duda si se está acentuando una concentración (y por ende una pérdida de bibliodiversidad) en la oferta de novedades que reciben las librerías" (Cámara Argentina del Libro, 2018, p. 27).

En este tema, Gloria Gómez Escalonilla (2004) se refiere a los problemas que la concentración vertical -por la cual las fusiones o adquisiciones se realizan en los distintos eslabones de la cadena de valor (Mastrini, 2014) - trae para la distribución de libros en España. Expone que la integración vertical de los grupos distribuidores por parte de las empresas editoriales beneficia la distribución de la producción propia del sello y perjudica a la ajena. En consecuencia, "las producciones de las pequeñas $\mathrm{y}$ medianas editoriales $\mathrm{y}$, por tanto, las producciones minoritarias y especializadas no se distribuyen o se distribuyen peor, y obviamente no llegan al mercado" (Gómez

${ }_{10}$ Djament plantea que desconoce el número exacto de la cantidad de librerías con las cuales trabaja. Esto se debe a que tal información la maneja la distribuidora. 
Gustavo Daniel Velázquez

Las editoriales independientes frente al problema de la distribución: Ios casos de Eterna Cadencia y Santiago Arcos

Escalonilla, 2004, p. 43). Si bien la autora se refiere a un caso europeo, el argumento se condice con la realidad de la industria editorial argentina.

La distribución mayorista tercerizada, entonces, tiene aspectos positivos para las editoriales independientes. Una empresa distribuidora puede contribuir a la circulación y a la visibilidad del catálogo del sello, ampliando sus posibilidades comerciales. Por ende, aporta significativamente al posicionamiento económico del proyecto. Pero las condiciones de mercado, altamente concentrado, ponen en desventaja a estos actores.

\section{Autodistribución}

Ante los problemas que genera la distribución tercerizada, algunos editores independientes deciden encarar ellos mismos esta tarea. La autodistribución se presenta como alternativa a la contratación de una distribuidora. La práctica genera ventajas respecto a la dependencia de un agente distribuidor a partir de una mayor autonomía en la organización de la circulación. Con base en esto, se despliegan ciertas condiciones que permiten la acción directa en la parte comercial.

En este método, el editor y la plantilla estable de trabajadores asumen los costos y el conjunto acciones de la circulación comercial.

En principio, asumir el trabajo de distribución permite retener el porcentaje que habitualmente se destina a un distribuidor externo como pago por sus servicios, el cual, en promedio, es de alrededor del 50\% del precio de venta al público del libro (Esteves, 2014). Este porcentaje lo comparte el agente distribuidor con el librero.

Al respecto, Gil y Gómez (2017) consideran que "el surgimiento continuo de pequeños editores que quieren llegar al mercado conlleva que los distribuidores apalanquen su fortaleza y posición competitiva frente a estos nuevos editores exigiendo porcentajes muy altos como comisión por la distribución" (p. 84). Por tanto, el capital que se reserva el sello resulta relevante para la economía general del proyecto.

El método de autodistribución, no obstante, implica una alta cuota de inversión para armar una estructura logística. Pueden mencionarse, entre otras cosas, los gastos de empleo de fletes, el armado y acondicionamiento de los depósitos y los materiales para la preparación de pedidos. Hacerse cargo de la distribución, además, supone resolver la parte administrativa. Para abarcar todos estos puntos se requieren altos niveles de capital, elemento que influye decisivamente a la hora de elegir el sistema de distribución adecuado para una editorial.

Villafañe considera que la autodistribución permite tener un seguimiento medido del rendimiento comercial de los libros. Por esa misma razón, solo puede manejarse con pocas librerías (la cantidad que le es posible abarcar).

Cuando le das [los libros] a un distribuidor, no tenés el control del stock ni de la cadena de pagos. En ese sentido, tenés que manejarte a ciegas porque no sabés si están o no están 


\begin{abstract}
los libros en las librerías, si se vendieron o no, si se cobraron o no, si te los pagan o no. En cambio, manejándote directamente con las librerías, tenés un control del stock de las librerías, cobrás cada treinta días y no cada noventa o ciento veinte o cuando le dé la gana al distribuidor. Tenés un dominio total del emprendimiento (Miguel Villafañe, editor de Santiago Arcos). ${ }^{11}$
\end{abstract}

Entonces, la distribución propia asegura la presencia de los libros en los puntos de venta. Esto significa, en el mayor de los casos, optimizar el saldo comercial del proyecto editorial. A partir de este método, es posible tener un seguimiento detallado del traslado, la colocación y las ventas de cada libro y, a la vez, un retorno económico más rápido dado que no hay mediadores entre la editorial y el librero (se acorta la cadena de pagos). De esta manera, se logra un monitoreo más preciso de la cantidad de librerías con las cuales se mantienen cuentas activas y de los libros que están en cada local.

La autodistribución, por tanto, lleva a conocer cómo funciona el canal librero. Es decir, el trabajo con las librerías se realiza de manera directa. Esto implica visitar los locales con regularidad para conocer la rotación de los títulos, informar al librero de las novedades o convencerlo de una exhibición en vidriera. Cuanto más informado esté el librero, mejor podrá recomendar la obra a posibles lectores. En este sentido, Djament reconoce que la editorial realiza un trabajo de seguimiento de determinadas librerías con las cuales existe una relación de confianza, complementando el trabajo de la distribuidora externa. En cuanto a esto, ambos editores coinciden en que la diferencia se logra a partir del trabajo personalizado y la comunicación fluida con los libreros, pues este vínculo acaba por influir en la suerte que tendrá el libro (Szpilbarg y Saferstein, 2012).

Tales razones llevan a pensar que si bien los circuitos comerciales son acotados, la estrategia resulta adecuada para el sostenimiento económico de Santiago Arcos. Como plantea Villafañe: "La editorial es autosuficiente. No hay excedente, es autosuficiente". Esto quiere decir que la distribución le permite al sello subsistir (saldar las cuentas de los proveedores, costear nuevas publicaciones) de acuerdo a la escala económica de este emprendimiento (distinta a la de Eterna Cadencia, que se maneja con una tirada promedio de mil quinientos ejemplares). Así, el sello financia al canal comercial: "La edición es financiada por los proveedores y nosotros, a su vez, financiamos a las librerías, a las cuentas corrientes".

Dados los desequilibrios que genera la distribución tercerizada a los cuales se ha hecho mención, la autodistribución parece ser la forma más adecuada para los sellos con fondos editoriales pequeños.

Sin embargo, el conjunto de tareas vinculadas a la distribución representa una cierta complejidad para proyectos que se sostienen por el esfuerzo de pocas personas, lo cual es característico de este tipo de plan editorial.

${ }^{11}$ Entrevista realizada el 15 de noviembre de 2017. 
Gustavo Daniel Velázquez

Las editoriales independientes frente al problema de la distribución: Ios casos de Eterna Cadencia y Santiago Arcos

La plantilla estable de Santiago Arcos es chica. Además de Villafañe, cuenta con un corredor de ventas que trabaja algunas horas a la semana y con trabajadores externos para muchas de las tareas de la producción editorial (corrección, diseño de interiores, diseño de cubiertas), por lo que el sello se aboca especialmente a la parte comercial, que se lleva adelante en gran medida desde la librería propia, espacio que también funciona como depósito.

Cuando una editorial no puede cubrir estas responsabilidades, puesto que insume muchas horas de trabajo, surge la necesidad de contratar personal que se aboque exclusivamente a la distribución. Sin embargo, son pocas las organizaciones que cuentan con las condiciones financieras necesarias para poder sumar trabajadores.

Santiago Arcos se sostiene por el esfuerzo de pocos integrantes -quienes cobran un sueldo-, lo que trae dificultades en cuanto al mantenimiento del proyecto, dado que su estructura no permite incorporar más personal que se aboque exclusivamente a la cuestión comercial. No obstante, el editor considera que el sistema de distribución propio es el más acorde para un proyecto editorial independiente:

La independencia del editor pasa por la distribución de sus libros, por tener el stock total a disposición y poder manejarlo. El editor que tiene los libros en una distribuidora creo que está en problemas y no es un editor independiente, o es más dependiente que otros (Miguel Villafañe, editor de Santiago Arcos). ${ }^{12}$

Respecto a la distribución, Djament adopta una perspectiva crítica de los modelos que se expusieron a lo largo de este trabajo. Considera que:

\footnotetext{
Ningún método tiene ventajas. La ventaja [de la distribución tercerizada] es que en parte te despreocupás del negocio, pero es mentira, no te despreocupás, porque son tus libros y estás velando permanentemente para que lleguen a todas las librerías posibles de Argentina. Pero me parece que los dos sistemas tienen ventajas y desventajas, no diría que es necesariamente mejor este (Leonora Djament, editora de Eterna Cadencia). ${ }^{13}$
}

Más allá de las diferencias en las posturas, ambos editores consideran la distribución como un aspecto transversal de sus proyectos editoriales; en definitiva, de todas las editoriales, cualquiera sea su tamaño o propuesta de publicación. Esto hace que la distribución sea un aspecto sensible que interfiere en las posibilidades de crecimiento de una empresa editorial.

\section{Conclusiones}

Los servicios de una distribuidora externa permiten a la editorial desligarse de la logística, la cual resulta costosa, además de implicar una significativa carga laboral. Sin

12 Entrevista realizada el 15 de noviembre de 2017.

13 Entrevista realizada el 22 de noviembre de 2017. 
embargo, las condiciones de la distribución privada no siempre resultan las más efectivas para las editoriales independientes, cuyas producciones son de rotación lenta y se orientan a segmentos especializados de la demanda.

Ante este problema, la autodistribución presenta una serie de beneficios para estos sellos, ya que se obtiene principalmente una mejor gestión del posicionamiento de los libros en los locales comerciales y, por ende, puede alcanzarse una mayor rentabilidad. Como contrapartida, no es menor el trabajo que significa para los gestores del sello. Se le agrega, también, el capital que se requiere para desarrollar una estructura logística. Por tanto, la autodistribución requiere una gran inversión y, sobre todo, una dedicación intensiva de los miembros de la editorial. Ambos fenómenos se encuentran enlazados.

A partir de los casos estudiados, pueden advertirse las variaciones en ambos métodos de distribución mayorista. Las complejidades que supone cada modelo ponen en tensión a las editoriales independientes que buscan colocar sus libros en librerías y llegar a la mayor cantidad posible de consumidores, por lo que optan por el método de circulación comercial que mejor se adecue a su realidad.

Eterna Cadencia ha crecido de la mano de la distribución externa, tanto en el plano nacional como en el internacional. Desde sus inicios, este sistema le ha sido redituable $y$, pese a que la editora reconoce que posee fallas, ha contribuido al posicionamiento del sello. No obstante, fortalece la distribución externa con la atención directa a librerías. Esto se asemeja a las prácticas propias de la autodistribución, lo que evidencia la importancia que le dan los editores a trabajar personalmente con los libreros para así acotar la intermediación entre ellos y fortalecer el emprendimiento, además de contribuir a la economía de la librería.

Por su parte, la autodistribución le ha permitido a Santiago Arcos sostenerse en el tiempo. Con circuitos comerciales acotados y distribución selectiva, este sello participa de la escena editorial. El saldo comercial que se obtiene a través de este método resulta acorde a la dimensión del proyecto. Si bien se logra mayor control de la circulación de los libros, como contrapartida la dedicación laboral resulta intensiva. Las tareas son absorbidas por el editor y se realizan a la par de otro tipo de trabajos de edición. En esta situación, en la medida en que el proyecto crezca, sería necesario incorporar personal, dado que no podría dar respuesta a la exigencia logística.

La distribución depende en gran medida de la escala económica de la empresa editorial. Esto hace que los grandes grupos posean una mayor atención de los distribuidores, lo que se traduce en una mayor dedicación en la colocación y seguimiento de los libros en los puntos de venta.

Esta condición afecta a toda la estructura de distribución nacional. En consecuencia, todos los sellos independientes deben esforzarse para adquirir visibilidad y hacerse conocidos dentro de la complejidad del sector. 
Gustavo Daniel Velázquez

Las editoriales independientes frente al problema de la distribución: Ios casos de Eterna Cadencia y Santiago Arcos

\section{Referencias}

Amadeo González, P. (2016). Relatos sobre trabajo en pequeñas editoriales. En Badenes, D. y Stedile Luna, V. (Comps.), Estado de Feria Permanente. La experiencia de las editoriales independientes argentinas 2001-2020 (pp. 63-84). La Plata: Club Hem Editores.

Badenes, D. (2019). La edición imperfecta. En Badenes, D. y Stedile Luna, V. (Comps.), Estado de Feria Permanente. La experiencia de las editoriales independientes argentinas 2001-2020 (pp. 21-44). La Plata: Club Hem Editores.

Becerra, M. y Mastrini, G. (2009). El problema de la concentración. En Los dueños de la palabra. Buenos Aires: Prometeo.

Becerra, M. y Mastrini, G. (2017). La concentración infocomunicacional en América Latina 2000-2015: nuevos medios y tecnologías, menos actores. Bernal: Universidad Nacional de Quilmes.

Bhaskar, M. (2014). La máquina de contenido. Hacia una teoría de la edición desde la imprenta hasta la red digital. México: Fondo de Cultura Económica.

Botto, M. (2006). La concentración y la polarización de la industria editorial. En Luis de Diego, J. (Dir.), Editores y políticas editoriales en Argentina 1880-2000. Buenos Aires: Fondo de Cultura Económica.

Bourdieu, P. (2000). Una revolución conservadora en la edición. En Intelectuales, política y poder. Buenos Aires: Eudeba.

Bustamante, E. (2008). Hacia un nuevo sistema mundial de comunicación: las industrias culturales en la era digital. Barcelona: Editorial Gedisa.

Cámara Argentina del Libro (2018). Informe de producción del libro argentino. Obtenido el 13 de mayo de 2020 de https://www.camaradellibro.com.ar/index. $\mathrm{php} /$ panorama-editorial/estadisticas

Cámara Argentina del Libro (2019). Informe de producción del libro argentino. Obtenido el 13 de mayo de 2020 de https://www.camaradellibro.com.ar/index. $\mathrm{php} /$ panorama-editorial/estadisticas

Centro de Estudios para la Producción (2014). La industria del libro en Argentina. Obtenido el 13 de mayo de 2020 de http://www.funcex.org.br/material/ redemercosul_bibliografia/biblioteca/ESTUDOS_ARGENTINA/ARG_51.pdf

Colleu, G. (2008). La edición independiente como herramienta protagónica de la bibliodiversidad. Buenos Aires: La Marca Editora.

Conde, J.y Hafter, L. (2019). Hacia una categoría de edición-arte. En Badenes, D. y Stedile Luna, V. (Comps.), Estado de Feria Permanente. La experiencia de las editoriales independientes argentinas 2001-2020 (pp. 21-44). La Plata: Club Hem Editores.

De Diego, J. L. (2006). Editores y políticas editoriales en Argentina 1880-2000. Buenos Aires: Fondo de Cultura Económica.

De Diego, J. L. (2012). Concentración económica, nuevos editores, nuevos agentes. 
Austral Gmunicación

Volumen 9, número 2 (Diciembre de 2020): 405-422. ISSN 2313-9129

Primer Coloquio Argentino de Estudios sobre el Libro y la Edición, La Plata. Obtenido el 13 de mayo de 2020 de http://www.memoria.fahce.unlp.edu.ar/trab_ eventos/ev.1930/ev.1930.pdf

De Diego, J. L. (2015). La otra cara de Jano. Una mirada crítica sobre el libro y la edición. Buenos Aires: Ampersand.

De Mateo Pérez, R., Saura, $\quad$ L. B. y Casals, M. S. (2009). Gestión de empresas de comunicación. Sevilla: Comunicación social.

Dujovne, A. (2017). La aventura intelectual de publicar un libro. Anfibia. Obtenido el 13 de mayo de 2020 de http://www.revistaanfibia.com/ensayo/ la-aventura-intelectual-de-publicar-un-libro/

Dujovne, A. (3 de marzo de 2018). Alejandro Dujovne: "La pérdida de diversidad editorial o de 'bibliodiversidad' conlleva, en definitiva, a un empobrecimiento de la vida cultural e intelectual de una sociedad". Revista Mutt. Obtenido el 13 de mayo de 2020 de http://revistamutt.com/letras/alejandro-dujovne/

Esteves, F. (2014). Manual de supervivencia para editores del siglo XXI. Buenos Aires: Eudeba.

Gil, M. y Gómez, M. (2017). Manual de edición: guía para estos tiempos revueltos. Buenos aires: La Marca Editora.

Gómez Escalonilla, G. (2004). La edición de libros: un sector potente a la defensiva digital. En Bustamante, E. (Comp.), Comunicación y cultura en la era digital: industrias, mercados y diversidad en España (pp. 37-66). Barcelona: Gedisa.

Jambeiro, O., Brittos, V. y Simis, A. (2005). Información y comunicaciones globales: para comprender el siglo XXI. En Bolaño, C., Mastrini, G. y Sierra F. (Comps.), Economía política, comunicación y conocimiento. Una perspectiva crítica latinoamericana (pp. 371-396). Buenos Aires: La Crujía.

López Winne, H. y Malumián, V. (2016). Independientes, ¿de qué? Hablan los editores de América Latina. México: Fondo de Cultura Económica.

Mastrini, G. (2014). Economía de la comunicación y la cultura. Bernal: Universidad Nacional de Quilmes.

Mosco, V. (2006). Economía Política de la Comunicación: una actualización diez años después. CIC. Cuadernos de Información y Comunicación, 11, 57-79.

Muniz Júnior, J. de S. (2015). Itinerarios de una identidad voluble: el debate sobre la edición. Orbis Tertius, 21, 145-158.

Páez, C. D. (2018). La concentración del mercado editorial en Argentina entre 1991 y 2001. (Tesis de posgrado). Bernal: Universidad Nacional de Quilmes. Obtenido el 13 de mayo de 2020 de http://ridaa.unq.edu.ar/handle/20.500.11807/909

Saferstein, E. (2013). La cocina del "best-seller" político: producción y circulación de géneros editoriales sobre la coyuntura socio-política argentina (2001-2011). (Tesis de posgrado). Universidad Nacional de San Martín. 
Gustavo Daniel Velázquez

Las editoriales independientes frente al problema de la distribución: Ios casos de Eterna Cadencia y Santiago Arcos

Saferstein, E. (2019). Las ferias de libros y sus públicos. Circulación, visibilidad y desigualdades estructurales en el campo editorial. En Badenes, D. y Stedile Luna, V. (Comps.), Estado de Feria Permanente. La experiencia de las editoriales independientes argentinas 2001-2020 (pp. 241-254). La Plata: Club Hem Editores.

Saferstein, E. y Szpilbarg, D. (2014). La industria editorial argentina 1990-2010: entre la concentración económica y la bibliodiversidad. Alternativa, 3, 1-21.

Schierloh, E. (junio de 2019). Sobre la independencia editorial (con coordenadas para evaluarla). Mímesis. Obtenido el 13 de mayo de 2020 de https://edicionesmimesis. cl/index.php/2019/06/19/sobre-la-independencia-editorial-con-coordenadaspara-evaluarla-por-eric-schierloh/

Szpilbarg, D. (2010). Editoriales artesanales y libros-arte: nuevos modos de producción y circulación social del libro. Reflexiones a partir del caso de las editoriales Funesiana y Clase Turista. VI Jornadas de Sociología de la UNLP, La Plata. Obtenido el 13 de mayo de 2020 de http://www.memoria.fahce.unlp.edu.ar/trab_eventos/ev.5755/ ev.5755.pdf

Szpilbarg, D. (2013). El editor como intermediario cultural. El caso de las editoriales digitales en Argentina. X Jornadas de Sociología, Facultad de Ciencias Sociales, Universidad de Buenos Aires, Buenos Aires, Argentina. Obtenido el 13 de mayo de 2020 de http://cdsa.aacademica.org/000-038/414.pdf

Szpilbarg, D. (2019). Cartografía Argentina de la edición Mundializada. Modos de hacer y pensar el libro en el siglo XXI. Buenos Aires: Tren en Movimiento.

Szpilbarg, D. y Saferstein, E. (2012). El espacio editorial "independiente": heterogeneidad, posicionamientos y debates. Hacia una tipología de las editoriales en el período 1998-2010. Primer Coloquio Argentino de Estudios sobre el Libro y la Edición, del 31 de octubre al 2 de noviembre. Universidad Nacional de La Plata, La Plata, Buenos Aires, Argentina.

Tremblay, G. (2011). Industrias Culturales, economía creativa y sociedad de la información. En Albornoz, L. (Comp.), Poder, Medios, Cultura. Una mirada crítica desde la economía política de la comunicación. Buenos Aires: Paidós.

Vanoli, H. y Saferstein, E. (2011). Cultura literaria e industria editorial. Desencuentros, convergencias y preguntas alrededor de la escena de las pequeñas editoriales. En Rubinich, L. y Miguel, P. (Eds.), Creatividad, economía y cultura en la ciudad de Buenos Aires 2001-2010 (pp. 1-10). Buenos Aires: Aurelia Rivera.

Velázquez, G, (2019). La distribución asociada: alianzas contingentes y proyectos asociados. En Badenes, D. y Stedile Luna, V. (Comps.), Estado de feria permanente. La experiencia de las editoriales independientes asociativas argentinas 2001-2020. La Plata: Club Hem Editores.

Zallo, R. (1988). Economía de la comunicación y la cultura. Madrid: Akal. 